HRJ

v.2 n.11 (2021)

Recebido: $25 / 10 / 2020$

Aceito: $27 / 04 / 2021$

\title{
Impacto econômico da atuação de equipes consultoras de Cuidados Paliativos inseridas em hospital
}

\section{Milena Lima dos Santos ${ }^{1}$ \\ Flávia Nunes Fonseca²}

\begin{abstract}
1Psicóloga. Graduada em Psicologia pela Unidade de Brasília. Residente do Programa de Residência Multiprofissional em Atenção ao Câncer (PRMAC), da Fundação de Ensino e Pesquisa em Ciências da Saúde (FEPECS), vinculada à Secretaria de Saúde do Distrito Federal (SES-DF).
\end{abstract}

2Psicóloga. Graduada em Psicologia pela Universidade de Brasília. Mestre em Ciências do Comportamento pela Universidade de Brasília. Especialista em Análise Comportamental Clínica pelo Instituto Brasiliense de Análise do Comportamento (IBAC). Psicóloga da Secretaria de Saúde do Distrito Federal (SES-DF), atuando no Serviço de Cuidados Paliativos do Hospital de Base do Distrito Federal.

\section{RESUMO}

Internações de pacientes crônicos são, em geral, prolongadas. Frequentemente empregam-se medidas inadequadas que, além de prolongar sofrimento, representam consumo de substanciais recursos de saúde. Nesse contexto, a atuação de equipes consultoras de Cuidados Paliativos (CP) pode representar incremento na qualidade do cuidado e redução dos custos. O objetivo deste estudo é apresentar os resultados de uma revisão bibliográfica sobre o impacto econômico da atuação de equipes consultoras de CP inseridas em hospitais durante o período de 2015 a 2020. Trata-se de uma revisão sistemática de literatura nos bancos de dados LILACS, SCOPUS (Elsevier), PubMed, SciELO e MEDLINE. Oito trabalhos atenderam aos critérios de inclusão. Há evidências de que intervenções das equipes consultoras de $\mathrm{CP}$ favorecem a redução de custos hospitalares, na medida em que contribuem para menores taxas de readmissão hospitalar, menor tempo médio de permanência, menor número de procedimentos diagnósticos e terapêuticos e menor número de admissões em UTI. Há vantagem econômica da abordagem de equipes consultoras de CP. Dessa forma, as evidências podem auxiliar na tomada de decisão de gestores de saúde quanto à organização de serviços e investimento em equipes especializadas em CP.

Palavras-chaves: Cuidado Paliativo, custos em saúde, equipe consultora

\section{Economic Impact of the Performance of Hospital Palliative Care Consultation Teams}

\begin{abstract}
Hospitalizations of chronic patients are, in general, prolonged. Inadequate measures are often used which, in addition to prolonging suffering, represent consumption of substantial health resources. In this context, the performance of palliative care (PC) consulting teams can represent an increase in the quality of care and a reduction in costs. The objective of this study is to present the results of a bibliographic review on the economic impact of the work of PC consulting teams inserted in hospitals during the period from 2015 to 2020 . This is a systematic review of the literature in the databases
\end{abstract}


LILACS, SCOPUS (Elsevier), PubMed, SciELO and MEDLINE. Eight studies met the inclusion criteria. There is evidence that interventions by PC consulting teams favor the reduction of hospital costs, as they contribute to lower rates of hospital readmission, shorter average length of stay, fewer diagnostic and therapeutic procedures and fewer ICU admissions. There is an economic advantage to the approach of PC consulting teams. Thus, the evidence can assist in the decision making of health managers regarding the organization of services and investment in teams specialized in PC.

Keywords: Palliative care; Health care costs; Consultation team

\section{INTRODUÇÃO}

No Brasil, a relevância de se ampliar a discussão acerca das condições crônicas se sustenta na necessidade de considerar a transição epidemiológica da população, devido ao surgimento de melhores recursos para diagnóstico e tratamento assim como melhora nos indicadores sociais e de saúdeํ. Diante disso, é possível dizer que o avanço tecnológico no ramo terapêutico promoveu a transformação de doenças antes tidas como mortais, em condições crônicas ${ }^{2 \cdot 3}$.

As internações de pacientes com condições crônicas são, por característica, prolongadas. Nelas, muitas intervenções passaram a ser utilizadas para a manutenção da vida. Entretanto, em relação aos pacientes com doenças incuráveis, frequentemente são empregadas medidas inapropriadas que podem trazer maior possibilidade de dano do que benefícios ${ }^{4}$. Nesses casos, além do prolongamento do sofrimento dos pacientes e familiares, nas internações prolongadas consomem-se substanciais recursos de saúde, especialmente, quando os pacientes são internados em Unidades de Terapia Intensiva e Semi - Intensiva, campos historicamente marcados pelo paradigma biomédico ${ }^{1,5}$.

Embora as internações de pacientes com condições crônicas resultem em altas despesas, os custos não significam qualidade de assistência em saúde. Observa-se que esses pacientes recebem baixa qualidade de assistência médica, caracterizada pelo tratamento inadequado de sintomas, necessidades não atendidas de cuidados pessoais, 
alta sobrecarga do cuidador e baixa satisfação do paciente e sua rede de suporte social em relação ao plano de cuidados ${ }^{6}$.

Por outro lado, o Manual de Cuidados Paliativos da Academia Nacional de Cuidados Paliativos (ANCP) traz a definição proposta pela Organização Mundial de Saúde (OMS):

"Cuidado Paliativo é uma abordagem que promove a qualidade de vida de pacientes e seus familiares, que enfrentam doenças que ameacem a continuidade da vida, através da prevenção e alívio do sofrimento. Requer a identificação precoce, avaliação e tratamento da dor e outros problemas de natureza física, psicossocial e espiritual" (Matsumoto, 2012, p. 26) ${ }^{7}$.

O hospital é o local de cuidado em saúde mais difundido e culturalmente aceito. Nesse sentido, apesar do movimento para expansão dos Cuidados Paliativos nos modelos ambulatorial e domiciliar, a predominância do cuidado hospitalocêntrico ainda é visível. Desse modo, o Cuidado Paliativo, em contexto hospitalar, pode ser realizado de três diferentes formas: em Unidade de Cuidados Paliativos (UCP); Equipe consultora ou volante ou pela Equipe itinerante ${ }^{8}$. Em uma UCP, o atendimento é conduzido por uma equipe especializada, cujo trabalho é pautado na filosofia dos Cuidados Paliativos. A equipe é responsável pelo acompanhamento de pacientes em um conjunto de leitos localizados em área determinada do hospital. A Equipe Consultora ou Volante, também especializada em Cuidados Paliativos, é acionada conforme a percepção da equipe de saúde assistente e avalia o paciente no setor em que estiver internado. Geralmente, essa equipe não assume completamente os cuidados com o paciente, mas atua como equipe de suporte, que discute e orienta condutas. Por último, a Equipe Itinerante é igualmente acionada conforme a avaliação do médico assistente, mas passa a assumir os cuidados 
no setor em que o paciente está internado, podendo o médico assistente dar continuidade ao acompanhamento em conjunto ou não ${ }^{8}$.

Os programas de Cuidados Paliativos hospitalares possibilitam melhor manejo físico e psicológico dos sintomas de pacientes com condições crônicas, favorecem o suporte ao cuidador, assim como promovem bem-estar e satisfação para as famílias desses pacientes ${ }^{6}$. Além disso, os Cuidados Paliativos nos hospitais estão associados a reduções significativas nos custos diários e nos custos totais das internações, e podem gerar economias substanciais no sistema de saúde por prevenção de custos ${ }^{9}$.

Nesse sentido, os Cuidados Paliativos hospitalares reduzem em até $40 \%$ os gastos no último mês de vida e em $17 \%$ nos últimos seis meses, quando comparados aos cuidados habituais ${ }^{10}$. Essa diminuição decorre, principalmente, em razão da redução de realização de exames diagnósticos e de procedimentos intervencionistas, assim como uso de Diretivas Antecipadas de Vontade ${ }^{11}$. As diretivas são os desejos que o paciente manifesta previamente no que se refere aos cuidados e tratamentos que deseja ou não receber quando estiver incapacitado de expressar, livre e autonomamente, sua vontade, em condições de doenças ameaçadoras à vida ${ }^{11}$. Dessa forma, uma vez que há possibilidade de o paciente opinar e expressar antecipadamente sobre o tipo de cuidados e tratamentos que gostaria de receber, há redução do número de internações em unidades de terapia intensiva, do uso de medidas invasivas e da realização de exames desnecessários.

No que se refere a Unidades de Cuidados Paliativos, verifica-se que existe redução dos custos hospitalares em comparação com demais unidades de internação. Há evidências de que os pacientes em Cuidados Paliativos não só apresentam menores custos, como menor frequência de internação em unidade de terapia intensiva, menor 
frequência de realização de procedimentos, menor permanência hospitalar e menor percentual de óbitos no hospital ${ }^{4}$.

Equipes consultoras de Cuidados Paliativos também estão associadas com economias de custos hospitalares significativos. Os resultados do estudo elaborado por Morrison et $a l^{6}$ publicado em 2008 apontam que, nos Estados Unidos, no tocante aos pacientes que foram acompanhados por equipes consultoras de Cuidados Paliativos e receberam alta, observou-se uma economia de US\$ 1696 em custos diretos por admissão e US\$ 279 em custos diretos por dia, incluindo reduções de custos em laboratório e unidade de terapia intensiva, em comparação com os pacientes de cuidados habituais. Já em relação aos pacientes que receberam o atendimento das equipes de Cuidados Paliativos e morreram, houve uma economia líquida ajustada de US\$ 4908 em custos diretos por admissão e US\$ 374 em custos diretos por dia, com reduções significativas em gastos relacionados à farmácia, laboratório e unidade de terapia intensiva, em comparação com os pacientes que receberam cuidados habituais ${ }^{6}$.

Um estudo realizado por Morrison et al ${ }^{12}$ publicado em 2011 investigou os impactos econômicos do atendimento prestado por uma equipe consultora em CP para pacientes inseridos no Medicaid, programa de saúde social dos Estados Unidos, em quatro hospitais do estado de Nova York. Os resultados demonstraram que os pacientes que receberam atendimento em Cuidados Paliativos da equipe consultora economizaram, em média, 6.900 dólares em custos hospitalares durante uma determinada admissão em comparação aos pacientes que receberam os cuidados usuais. Essas reduções incluíram 4.098 dólares em custos hospitalares por admissão para pacientes que receberam alta com vida e 7.563 dólares para pacientes que morreram no hospital. Considerando as indicações médicas e a concordância de pacientes e suas famílias, os pacientes que receberam atendimento em Cuidados Paliativos são menos 
propensos a morrer em unidades de terapia intensiva, e mais propensos a serem referenciados para hospices e ambulatórios de Cuidados Paliativos, em comparação aos pacientes que receberam cuidados usuais ${ }^{12}$.

Para além dos ganhos relativos à economia de custos, aponta-se como vantagem da equipe consultora, em relação às demais modalidades de assistência em Cuidados Paliativos, o potencial de disseminar rapidamente a filosofia de cuidado de final de vida, haja vista que, ela está em contato frequente com várias alas e equipes do hospital, e possui assim papel educador em relação aos demais profissionais da assistência ${ }^{7}$.

Os dados apresentados ilustram como a avaliação econômica na assistência à saúde é fundamental para embasar a gestão de políticas públicas e alocação de recursos, com o objetivo de favorecer o cuidado dos pacientes com condições crônicas sem indicação de tratamentos modificadores de doença. Diante do exposto, o presente artigo objetiva apresentar os resultados de uma revisão bibliográfica das evidências nacionais e internacionais disponíveis sobre o impacto econômico resultante da atuação de equipes consultoras de Cuidados Paliativos inseridas em hospitais, durante o período de 2015 a 2020. Nesse sentido, visa contribuir com a desmistificação dos Cuidados Paliativos, demonstrando possíveis vantagens da inserção desse tipo de atendimento em hospitais de modo a fortalecer o cuidado aos pacientes com doenças que ameaçam a vida, bem como fornecer elementos que possam subsidiar a elaboração de políticas públicas de saúde.

\section{MÉTODO}

Trata-se de uma revisão sistemática de literatura. Para a elaboração da revisão foram seguidas as seguintes etapas: (1) definição da pergunta de pesquisa; (2) definição dos descritores para a busca; (3) definição dos critérios de elegibilidade dos artigos; (4) 
realização da pesquisa nas bases de dados; (5) definição das informações a serem extraídas dos estudos selecionados; (6) seleção dos artigos para compor a revisão a partir de triagem dos títulos e resumos utilizando critérios de inclusão e exclusão préespecificados; (7) leitura na íntegra dos estudos selecionados pelos títulos e resumos; (8) extração dos dados e informações de acordo com os objetivos da pesquisa com preenchimento de planilha criada para este fim; (9) síntese das evidências; (10) interpretação dos resultados e síntese do conhecimento produzido ${ }^{4}$.

Para nortear o estudo, formulou-se a questão: "Qual o impacto econômico da atuação de equipes consultoras de Cuidados Paliativos inseridas em hospitais?".

Estratégia de Busca

Os seguintes Descritores em Ciências de Saúde (DeCS) foram inseridos na busca: "palliative care", "health care costs" e "consultation team". Os descritores foram inseridos nas plataformas de busca com a expressão booleana "and". Diante da conhecida indexação de publicações na área da saúde, os bancos de dados utilizados foram os bancos de dados da Literatura Latino-Americana e do Caribe em Ciências da Saúde (LILACS), SCOPUS (Elsevier), PubMed, SciELO (Scientific Electronic Library Online) e Medical Literature Analysis and Retrieval System Online (MEDLINE), sendo esta última acessada através da Biblioteca Virtual de Saúde (BVS).

Para registro das informações-chave, foi elaborada e preenchida uma planilha que permitiu reunir e organizar elementos referentes à autoria e publicações (base de dados de indexação, título, ano, autor, país de origem, idioma, periódico, modelo do estudo, objetivos e principais resultados).

Critérios de Elegibilidade

Dos critérios de inclusão, a estratégia de seleção se limitou a artigos originais (publicações com resultados primários de pesquisa científica, apresentando dados 
originais de estudos experimentais ou observacionais, com análise descritiva ou inferencial de dados próprios); escritos em português ou inglês; publicados no período de 2015 a 2020; envolvendo pacientes adultos hospitalizados, de qualquer sexo; pesquisas sobre custos em saúde envolvendo pacientes recebendo Cuidados Paliativos através de equipes consultoras, em comparação com os pacientes de cuidados habituais. A busca se limitou aos últimos cinco anos devido à rápida mudança das análises econômicas e desenvolvimento científico em saúde.

Quanto aos critérios de exclusão, a aplicação dos critérios de pesquisa aos bancos de dados forneceu uma lista para triagem de títulos. Os títulos foram excluídos com base em alguns critérios: enfoque farmacológico; artigos sob formas de resumo e trabalhos publicados em anais de congressos; estudos publicados como capítulos de livro; grupos de comparação compostos por pacientes em Cuidados Paliativos domiciliares ou ambulatoriais; artigos sem foco em Cuidados Paliativos e nenhum foco específico em custos ou utilização de serviços de saúde.

Seleção dos Artigos

Realizou-se a leitura dos títulos dos artigos oriundos da busca. Após a seleção pelos títulos, os resumos foram lidos com o objetivo de verificar se preenchiam os critérios de inclusão. Após isso, os artigos selecionados foram lidos na íntegra e, em seguida, foram retiradas as informações-chave pré-determinadas, considerando a questão de investigação inicial. Posterior ao preenchimento da planilha de resumo de dados foi realizada uma análise temática do texto a partir da observação dos resultados referentes ao impacto econômico das equipes consultoras, a partir da estimativa de custos em relação às intervenções. 


\section{RESULTADOS E DISCUSSÕES}

A busca inicial nas bases de dados resultou em 84 artigos. Com a aplicação dos filtros representados pelos limites temporais e de restrição de idiomas (português e inglês), o total passou para 36. Dessa forma, os artigos foram inseridos na planilha do aplicativo Excel, de acordo com a base de indexação, a partir da ordem alfabética dos títulos, com o registro dos autores, e assim, sendo possível excluir os artigos em duplicidade. Nesta segunda filtragem, foram excluídos 13 artigos. A partir da leitura dos resumos, foi realizada avaliação considerando os critérios de inclusão e exclusão, de forma que foram selecionados 11 artigos. Por meio da leitura integral destes artigos, excluíram-se 3 por não responderem à pergunta norteadora, restando assim, 8 artigos (Figura 1). 


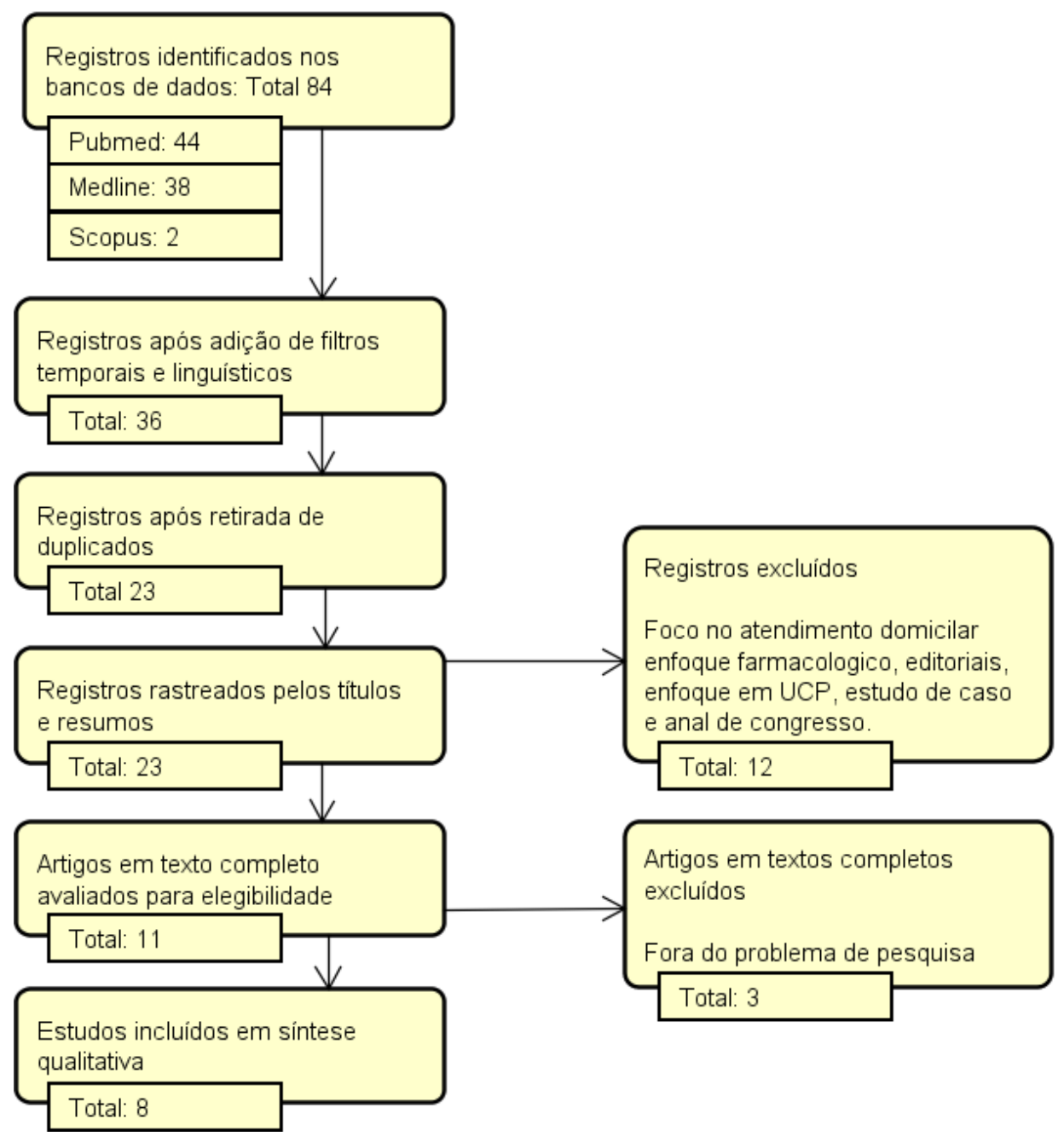

Figura 1. Fluxograma para resumo do processo de busca e inclusão/exclusão das publicações inseridas na revisão. Brasília, DF, Brasil, 2020.

Todos os artigos incluídos na seleção foram publicados no idioma inglês. Em relação à base de dados, a grande maioria foi encontrada por meio da MEDLINE (5 artigos). Na observação das características metodológicas das publicações selecionadas, encontrou-se que sete foram estudos de modelo observacional e um estudo clínico randomizado controlado; três artigos descreveram estudos retrospectivos e cinco, estudos prospectivos. Todos os estudos analisados receberam aprovação do comitê de ética em pesquisa institucional. 
O Quadro 1 abaixo contém o detalhamento das informações centrais dos oito artigos encontrados nesta revisão.

Quadro 1: Detalhamento dos artigos encontrados.

\begin{tabular}{|c|c|c|c|c|c|c|}
\hline Base de Dados & Título / Referência & Ano & País & Modelo do Estudo & Objetivos & Principais Resultados \\
\hline PUBMED & $\begin{array}{lr}\text { Association } & \text { between } \\
\text { Palliative Care and } & \text { End-of-Life } \\
\text { Resource Use for Older Adults } \\
\text { Hospitalized with Septic Shock. }{ }^{13}\end{array}$ & 2018 & EUA & $\begin{array}{l}\text { Estudo de coorte } \\
\text { retrospectivo. }\end{array}$ & $\begin{array}{l}\text { Determinar se os cuidados paliativos em regime de } \\
\text { internação, prestados por equipes consultoras } \\
\text { especializadas ou equipe médica primária, estão } \\
\text { associados à redução da permanência hospitalar e aos } \\
\text { custos para idosos com choque séptico no final da vida. }\end{array}$ & $\begin{array}{l}\text { Os cuidados paliativos foram associados a menor tempo } \\
\text { médio de permanência hospitalar, custos hospitalares } \\
\text { totais mais baixos e menor custo hospitalar diário, em } \\
\text { comparação a pacientes que não receberam o } \\
\text { atendimento da equipe especializada. }\end{array}$ \\
\hline MEDLINE & $\begin{array}{l}\text { Cost analysis of a prospective multi- } \\
\text { site cohort study of palliative care } \\
\text { consultation teams for adults with } \\
\text { advanced cancer: Where do cost- } \\
\text { savings come from? }^{14}\end{array}$ & 2017 & EUA & $\begin{array}{l}\text { Estudo de coorte } \\
\text { prospectivo em vários } \\
\text { locais. }\end{array}$ & $\begin{array}{l}\text { Analisar as diferenças de custo associadas às equipes } \\
\text { consultoras de cuidados paliativos usando duas perguntas } \\
\text { de pesquisa: (Q1) Qual é a associação entre a intervenção } \\
\text { precoce da equipe consultora de cuidados paliativos e a } \\
\text { intensidade dos tratamentos e tempo de permanência, em } \\
\text { comparação apenas com os cuidados habituais. (Q2) Qual } \\
\text { é a associação entre a intervenção precoce da equipe de } \\
\text { consultora em cuidados paliativos e os custos hospitalares } \\
\text { diários. }\end{array}$ & $\begin{array}{l}\text { A economia de custos dos cuidados paliativos precoces } \\
\text { ocorre devido à redução do tempo de internação e da } \\
\text { intensidade do tratamento, com uma estimativa de } 63 \% \\
\text { da economia associada ao menor tempo de internação. } \\
\text { Uma redução nos custos diários é observável nos dias } \\
\text { imediatamente após a consulta inicial, mas não persiste } \\
\text { indefinidamente. }\end{array}$ \\
\hline PUBMED & $\begin{array}{l}\text { Early Palliative Care Consultation in } \\
\text { the Medical ICU: A Cluster } \\
\text { Randomized Crossover Trial. }{ }^{15}\end{array}$ & 2019 & EUA & $\begin{array}{l}\text { Estudo clínico } \\
\text { randomizado } \\
\text { controlado } \\
\text { prospectivo. }\end{array}$ & $\begin{array}{l}\text { Avaliar o impacto da consulta precoce de Cuidados } \\
\text { Paliativos nas intervenções realizadas em pacientes de } \\
\text { alto risco de mortalidade internados em Unidades de } \\
\text { Terapia Intensiva (UTI), em comparação a aqueles que } \\
\text { receberam cuidados habituais. }\end{array}$ & $\begin{array}{l}\text { A indicação para não reanimar e não intubar ocorreu } \\
\text { mais cedo e significativamente mais frequentemente no } \\
\text { grupo de intervenção do que no grupo controle }(50,5 \% \\
\text { vs } 23,4 \% \text {; p <0,0001). O grupo intervenção teve } \\
\text { significativamente mais planos terapêuticos voltados } \\
\text { para cuidados paliativos ( } 18,6 \% \text { vs } 4,9 \% \text {; }<0,01) \text { com } \\
\text { menos dias de ventilação (mediana } 4 \text { vs } 6 \mathrm{~d} \text {; p <0,05), } \\
\text { menos traqueostomias realizadas ( } 1 \% \text { vs } 7,8 \% \text {; p }\end{array}$ \\
\hline
\end{tabular}




\begin{tabular}{|c|c|c|c|c|c|c|}
\hline & & & & & & $\begin{array}{l}<0,05) \text {, e menos visitas e / ou reinternações em serviços } \\
\text { de emergência pós-alta }(17,3 \% \text { vs } 38,9 \% \text {; } p<0,01) \text {. }\end{array}$ \\
\hline MEDLINE & $\begin{array}{l}\text { Hospital palliative care consult } \\
\text { improves value-based purchasing } \\
\text { outcomes in a propensity score- } \\
\text { matched cohort }^{16}\end{array}$ & 2019 & EUA & $\begin{array}{l}\text { Estudo de caso } \\
\text { controle. }\end{array}$ & $\begin{array}{l}\text { Compreender como uma consulta de Cuidados Paliativos } \\
\text { feita por equipe consultora afeta as taxas de readmissão de } \\
\text { pacientes e infecções adquiridas em hospitais, associadas } \\
\text { a métricas de custo-efetividade. }\end{array}$ & $\begin{array}{l}\text { Ao esclarecer o prognóstico e as metas do paciente, a } \\
\text { consulta de Cuidados Paliativos reduz as taxas de } \\
\text { readmissão. Em relação às infecções adquiridas no } \\
\text { hospital, foram semelhantes ou aumentaram em } \\
\text { comparação com os controles correspondentes. Sugere-se } \\
\text { que, isso se deva ao grau de complexidade dos pacientes. }\end{array}$ \\
\hline MEDLINE & $\begin{array}{l}\text { Integration of Palliative Care } \\
\text { Advanced Practice Nurses Into } \\
\text { Intensive Care Unit Teams }{ }^{17}\end{array}$ & 2017 & EUA & $\begin{array}{l}\text { Estudo de caso } \\
\text { controle. }\end{array}$ & $\begin{array}{l}\text { Avaliar a associação entre a integração dos enfermeiros } \\
\text { paliativistas nas operações rotineiras das UTIs e os custos } \\
\text { hospitalares em } 2 \text { instituições diferentes. Foi avaliada a } \\
\text { relação entre um modelo colaborativo de atuação da } \\
\text { equipe consultora e a redução de custos hospitalares. }\end{array}$ & $\begin{array}{l}\text { Os custos hospitalares de internação na instituição } 1 \\
\text { foram significativamente menores para pacientes com } \\
\text { cuidados paliativos em comparação com aqueles que não } \\
\text { receberam cuidados paliativos (mediana = US \$ } 6643 \text { vs } \\
\text { US \$ } 12 \text { 399, P <0,001). As diferenças de custo para } \\
\text { pacientes de UTI com e sem cuidados paliativos na } \\
\text { instituição } 2 \text { não foram significativamente diferentes. }\end{array}$ \\
\hline MEDLINE & $\begin{array}{l}\text { Palliative Care Teams' Cost-Saving } \\
\text { Effect Is Larger For Cancer Patients } \\
\text { With Higher Numbers Of }^{\text {Comorbidities }}{ }^{18}=\end{array}$ & 2016 & EUA & $\begin{array}{l}\text { Estudo de coorte } \\
\text { prospectivo em } \\
\text { vários locais. }\end{array}$ & $\begin{array}{l}\text { Avaliar o impacto da consulta de Cuidados Paliativos } \\
\text { sobre custos hospitalares para adultos com câncer } \\
\text { avançado, excluindo aqueles com demência, a partir da } \\
\text { atuação de equipes consultoras. }\end{array}$ & $\begin{array}{l}\text { Em comparação aos cuidados usuais, o recebimento de } \\
\text { uma consulta de CP dentro de dois dias da admissão foi } \\
\text { associada a custos menores para pacientes com maiores } \\
\text { números de comorbidades. Consultas precoces de CP } \\
\text { também foram associadas a um maior efeito de economia } \\
\text { de custos para todas as subamostras definidas por } \\
\text { multimorbidade. }\end{array}$ \\
\hline MEDLINE & $\begin{array}{l}\text { Prospective Cohort Study of Hospital } \\
\text { Palliative Care Teams for Inpatients } \\
\text { With Advanced Cancer: Earlier }\end{array}$ & 2015 & EUA & $\begin{array}{l}\text { Estudo de coorte } \\
\text { prospectivo em } \\
\text { vários locais. }\end{array}$ & $\begin{array}{l}\text { Avaliar se o tempo da consulta de cuidados paliativos, } \\
\text { após a admissão, tem impacto nos custos hospitalares. }\end{array}$ & $\begin{array}{l}\text { Consultas precoces de CP estão associadas a um efeito } \\
\text { maior nos custos hospitalares. Estima-se que, a } \\
\text { intervenção dentro de } 6 \text { dias, reduz os custos em US \$ }\end{array}$ \\
\hline
\end{tabular}




\begin{tabular}{|c|c|c|c|c|c|c|}
\hline & $\begin{array}{l}\text { Consultation Is Associated With } \\
\text { Larger Cost-Saving Effect. }^{19}\end{array}$ & & & & & $\begin{array}{l}1.312 \text { (IC } 95 \% \text {, US \$ - } 2.568 \text { a US \$ 56; P .04) em } \\
\text { comparação com não ter tido } \\
\text { intervenção, e intervenção em } 2 \text { dias por US \$ - } 2.280 \text { (IC } \\
95 \% \text {, US \$ - } 3.438 \text { a US \$ - 1.122; P .001); } \\
\text { essas reduções são equivalentes a uma redução de } 14 \% \text { e } \\
24 \% \text {, respectivamente, no custo da internação hospitalar. }\end{array}$ \\
\hline PUBMED & $\begin{array}{l}\text { The association between palliative } \\
\text { care team consultation and hospital } \\
\text { costs for patients with advanced } \\
\text { cancer: An observational study in } 12 \\
\text { Dutch hospitals }{ }^{20}\end{array}$ & 2019 & Holanda & $\begin{array}{l}\text { Estudo de coorte } \\
\text { prospectivo em } \\
\text { vários locais. }\end{array}$ & $\begin{array}{l}\text { Investigar a associação entre a consulta da equipe } \\
\text { consultora de Cuidados Paliativos e os custos dos } \\
\text { cuidados hospitalares em pacientes com câncer avançado. }\end{array}$ & $\begin{array}{l}\text { Análises usando escores de propensão não mostraram } \\
\text { diferença significativa nos custos hospitalares entre } \\
\text { pacientes que receberam CP e aqueles que receberam } \\
\text { cuidados habituais. }\end{array}$ \\
\hline
\end{tabular}

Fonte: elaborado pelas autoras. 
A revisão indicou estudos com desenhos metodológicos com significativa heterogeneidade. Dessa forma, foi feita uma análise temática dos resultados, a partir da estimativa de custos em relação à intervenção de equipes consultoras de Cuidados Paliativos. Assim, foi possível estabelecer quatro categorias nas quais os artigos se ajustavam.

Categoria 1: Nesta categoria estão estudos em que o impacto econômico das intervenções de equipes consultoras de Cuidados Paliativos significa redução de custos oriunda de menores taxas de readmissão hospitalar, menor tempo médio de permanência, menor número de procedimentos diagnósticos e terapêuticos e menor número de admissão em UTI (3 artigos) ${ }^{13,16,17}$;

Categoria 2: Nesta categoria está um estudo que avaliou o impacto econômico das intervenções de uma equipe consultora em $\mathrm{CP}$ considerando o número de comorbidades dos pacientes. Os resultados sugerem que há maior redução de custos para pacientes com câncer avançado com maior número de comorbidades, em comparação com aqueles com câncer avançado com menor número de comorbidades, devido à redução no número de exames e tempo de permanência $(1 \text { artigo })^{18}$.

Categoria 3: Nesta categoria estão estudos em que o impacto econômico das intervenções de equipes consultoras de CP foi avaliado levando-se em consideração a precocidade do encaminhamento para atendimento com equipe consultora em $\mathrm{CP}$. Verificou-se que quanto mais precoce o encaminhamento, maior a redução de custos (3 $\operatorname{artigos})^{14,15,19}$;

Categoria 4: Nesta categoria está um estudo em que não se demonstrou relação entre redução de custos e intervenções de equipes consultoras de Cuidados Paliativos realizadas no ambiente hospitalar $(1 \text { artigo })^{20}$. 
Os oito estudos foram realizados em países desenvolvidos, quase todos nos Estados Unidos (EUA). Apenas um dos estudos foi intervencionista ${ }^{15}$. Os resultados desse estudo apontam que, o grupo que recebeu o acompanhamento com equipe consultora de Cuidados Paliativos, teve maior número de desospitalizações, pacientes com menos dias em ventilação mecânica, menos traqueostomias realizadas, e menos visitas e/ou reinternações em serviços de emergência pós-alta. Os achados mostram que, embora o custo operacional total não tenha sido significativamente diferente entre os grupos controle e intervenção, os custos operacionais da UTI médica e da farmácia foram significativamente menores no grupo que recebeu as intervenções da equipe consultora.

Os demais artigos foram observacionais, dessa forma, uma relação de causa e efeito não pode ser definida. No entanto, há resultados que sugerem que o recebimento de Cuidados Paliativos por equipes consultoras está associado à menor tempo de permanência e a menores custos hospitalares totais e diários ${ }^{13}$, considerando que, quando são precoces, reduzem a intensidade do tratamento, no sentido de reduzirem a indicação de tratamentos fúteis e exames desnecessários ${ }^{14,16,19}$.

Além disso, também foram descritas evidências que sugerem que ao esclarecer o prognóstico e as metas do tratamento ao paciente, o atendimento das equipes consultoras de cuidados paliativos reduz as taxas de readmissão ${ }^{16}$. Os resultados do estudo de O’Mahony $\mathrm{S}$, et $\mathrm{al}^{17}$, sugerem ainda que, além do encaminhamento precoce, a busca ativa de pacientes com critérios para Cuidados Paliativos, favorece a economia de custos hospitalares.

Por definição Cuidado Paliativo é uma abordagem voltada para pacientes e seus familiares, que enfrentam doenças que ameacem a continuidade da vida ${ }^{7}$, nesse sentido, entende-se que é benéfico para todos os pacientes nessa condição. Entretanto, diante da 
escassez de profissionais qualificados, caso seja necessário estabelecer critérios de prioridade de atendimento, observou-se que os Cuidados Paliativos especializados são mais econômicos em pacientes com mais comorbidades e, portanto, devem ser $\operatorname{priorizados}^{18}$

Conforme apontado anteriormente, apenas o estudo de Brinkman-Stoppelenburg et al. ${ }^{20}$ não encontrou evidências de um efeito da atuação de equipes consultoras em Cuidados Paliativos sobre os custos hospitalares. A pesquisa fez uma comparação entre pacientes que foram atendidos por equipes consultoras e pacientes que não tiveram essa avaliação em 12 hospitais holandeses. Primeiramente, observou-se que os pacientes do primeiro grupo tinham expectativa de vida menor, performance status pior e, em sua maioria, não tinham mais proposta de tratamento modificador de doença. A análise demonstrou que o tempo de internação, as admissões em UTI, bem como o uso de procedimentos diagnósticos, medicações, alimentação por sonda e equipamentos para suporte respiratório foi similar entre os dois grupos, não havendo assim diferença significativa no tocante aos custos hospitalares. A hipótese levantada pelos autores para a compreensão deste dado, que difere de outras evidências documentadas na literatura, é a de que a intervenção das equipes consultoras de Cuidados Paliativos no cenário holandês frequentemente está acontecendo tardiamente considerando a trajetória da doença dos pacientes. Dessa maneira, em consonância com o que foi descrito na literatura, as conclusões deste artigo reforçam a importância de que tal intervenção aconteça mais precocemente, tanto pelo benefício direto à qualidade do cuidado oferecido aos pacientes, como para que se possa obter economia de recursos. Discutiuse ainda a possibilidade de que o prognóstico ruim dos pacientes seja uma variável confundidora, já que a avaliação de equipes consultoras de Cuidados Paliativos 
frequentemente é solicitada para pacientes com limitada expectativa de vida, o que já pode afetar por si só o uso de recursos hospitalares.

Vale destacar que, nenhum dos artigos encontrados foi realizado em países latinos e/ou em países em desenvolvimento, demonstrando que são necessários mais estudos acerca do impacto econômico dos Cuidados Paliativos nesses contextos, considerando suas especificidades. Outro ponto a ser destacado, é que não é facilmente identificada nos artigos a composição das equipes consultoras de Cuidados Paliativos. Além disso, a maioria dos estudos aponta apenas sobre o impacto da intervenção de equipes consultoras de Cuidados Paliativos nos custos hospitalares. Não está clara a relação entre o impacto econômico das equipes consultoras e o conteúdo das intervenções, por exemplo, se as intervenções eram reuniões familiares e/ou acolhimentos.

\section{CONCLUSÕES}

Pacientes com condições crônicas são responsáveis por uma alta proporção de gastos com saúde e considerando a transição epidemiológica da população brasileira, o presente estudo objetivou apresentar os resultados de uma revisão bibliográfica das evidências nacionais e internacionais disponíveis sobre o impacto econômico resultante da atuação de equipes consultoras de Cuidados Paliativos inseridas em hospitais. Nesse sentido, os estudos incluídos nesta revisão apontam que, as intervenções em Cuidados Paliativos têm impacto econômico nos custos hospitalares, significando redução de custos dos cuidados, em comparação às intervenções hospitalares habituais.

Espera-se que esses resultados auxiliem na tomada de decisão à medida que gestores busquem melhorar o atendimento para pacientes com doenças ameaçadoras de vida, aumentando o número de equipes qualificadas, enquanto reduzem os custos. Além 
disso, que esse referencial favoreça a compreensão dos Cuidados Paliativos, enquanto abordagem ampla, que reduz custos por favorecer o cuidado individualizado.

Uma limitação importante deste estudo é o fato de o mesmo não ter contemplado produções no idioma espanhol, devido aos critérios de inclusão. Além disso, não foram encontradas produções no contexto brasileiro, considerando o objetivo de destacar evidências nacionais e internacionais disponíveis sobre o impacto econômico resultante da atuação de equipes consultoras de Cuidados Paliativos inseridas em hospitais. Sugerem-se novos estudos acerca da temática em hospital brasileiro para que seja possível conhecer as especificidades desse contexto considerando as particularidades deste país.

\section{AGRADECIMENTOS}

Agradecemos toda a equipe do Serviço de Cuidados Paliativos (SECPA) do Hospital de Base do Distrito Federal (HBDF) pelo suporte oferecido durante a elaboração deste estudo.

\section{REFERÊNCIAS}

1. Moreira MCN, Gomes R \& Sá MRCD. Doenças crônicas em crianças e adolescentes: uma revisão bibliográfica. Ciência \& Saúde Coletiva 2014, 19(7), 20832094. [acessado 2020 Jul $31]$. Disponível em: <https://scielosp.org/article/csc/2014.v19n7/2083-2094/>.

2. Agar M, Phillips JL. Palliative medicine and care of the elderly. In: Cherny N, Fallon M, Kaasa S, Portenoy RK, Currow DC (Eds.), Oxford Textbook of Palliative Medicine. 5 ed. Oxford University Press; $2015 . \quad$ p. 1044-56. DOI:10.1093/med/9780199656097.003.0163. [acessado 2020 Ago 31]. Disponível 
em:<https://oxfordmedicine.com/view/10.1093/med/9780199656097.001.0001/med9780199656097>

3. Payne S, Lynch $\mathrm{T}$. International progress in creating palliative medicine as a specialized discipline and the development of palliative care. In: Cherny N, Fallon M, Kaasa S, Portenoy RK, Currow DC (Eds.), Oxford Textbook of Palliative Medicine. 5 ed. Oxford University Press; 2015. p. 3-9. DOI: 10.1093/med/9780199656097.003.0001. [acessado 2020 Ago 31]. Disponível em: <https://oxfordmedicine.com/view/10.1093/med/9780199656097.001.0001/med$9780199656097>$.

4. de-Vasconcelos T. Economic Impact Of Hospital Paliative Care Units: A Systematic Review / Impacto Econômico das Unidades de Cuidados Paliativos Hospitalares: Revisão Sistemática. Revista de Pesquisa: Cuidado é Fundamental Online [Internet]. 2019 Dec;11(5):1389-1396. [acessado 2020 Jul 31]. Disponível em: <http://www.seer.unirio.br/index.php/cuidadofundamental/article/view/9439/pdf $>$.

5. Moritz RD, Rossini JP, Deicas A. Cuidados Paliativos na UTI: definições e aspectos éticos e legais. In: Moritz RD (Org.). Cuidados Paliativos nas Unidades de Terapia Intensiva. São Paulo: Editora Atheneu; 2012. p. 19-32.

6. Morrison RS, Penrod JD, Cassel JB, et al. Cost savings associated with US hospital palliative care consultation programs. Arch Intern Med 2008 Sep;168(16):1783-90. [acessado 2020 Jul 31]. Disponível em: <https://pubmed.ncbi.nlm.nih.gov/18779466/>.

7. Matsumoto DY. Cuidados Paliativos: conceitos, fundamentos e princípios. Em Carvalho, RTD. \& Parsons, HA (Org.). Manual de cuidados paliativos ANCP. Ampliado e atualizado. 2 ed. Academia Nacional de Cuidados Paliativos; 2012. p. 23- 
30. [acessado 2020 Jul $31]$. Disponível em: $\langle$ https://dms.ufpel.edu.br/static/bib/manual_de_cuidados_paliativos_ancp.pdf $>$.

8. Rodrigues LF. Modalidades de atuação e modelos de assistência em Cuidados Paliativos. Em Carvalho RTD \& Parsons HA (Org..). Manual de cuidados paliativos ANCP. Ampliado e atualizado. 2 ed. Academia Nacional de Cuidados Paliativos; 2012, p. 86 - 93. [acessado 2020 Jul 31]. Disponível em: 〈https://dms.ufpel.edu.br/static/bib/manual_de_cuidados_paliativos_ancp.pdf $>$.

9. Smith TJ, Cassel JB. Cost and non-clinical outcomes of palliative care. J Pain Symptom Manage 2009 Jul;38(1):32-44. [acessado 2020 Jul 31]. Disponível em: <https://pubmed.ncbi.nlm.nih.gov/19615625/>.

10. Ribeiro SZRS, Vidal SA, Oliveira AG, et al. Custos e qualidade de vida de pacientes em cuidados paliativos. Rev enferm UFPE online 2018 Jun;12(6):1688-95 [acessado 2020 Jul 31]. Disponível em: <https://webcache.googleusercontent.com/search?q=cache:4f1_IeuGFWAJ:https://perio dicos.ufpe.br/revistas/revistaenfermagem/article/download/234832/29213+\&cd=1\&hl= pt-BR\&ct=clnk\&gl=br $>$.

11. Conselho Federal de Medicina. Resolução número 1.995, de 2012. [acessado 2020 Jul 31]. Disponível em: 〈https://www.legisweb.com.br/legislacao/?id=244750>.

12. Morrison RS, et al. Palliative care consultation teams cut hospital costs for Medicaid beneficiaries. Health Aff (Millwood) 2011 Mar;30(3):454-63. acessado 2020 Jul 31]. Disponível em: 〈https://pubmed.ncbi.nlm.nih.gov/21383364/>.

13. Maley JH, Worsham CM, Landon BE, Stevens JP. Association between Palliative Care and End-of-Life Resource Use for Older Adults Hospitalized with Septic Shock. Ann Am Thorac Soc 2020 Aug;17(8):974-979. [acessado 2020 Agos 10]. 
Disponível em: <https://www.atsjournals.org/doi/abs/10.1513/AnnalsATS.2020010380C>.

14. May P, Garrido MM, Cassel JB, et al. Cost analysis of a prospective multi-site cohort study of palliative care consultation teams for adults with advanced cancer: where do cost-savings come from? Palliat Med 2017 Apr;31(4):378-386. [acessado 2020 Agos $11]$.

Disponível

em: <https://journals.sagepub.com/doi/10.1177/0269216317690098 >

15. Ma J, Chi S, Buettner B, et al. Early palliative care consultation in the medical ICU: A cluster randomized crossover trial. Crit Care Med 2019 Dec;47(12):1707-1715 [acessado 2020 Agos 10]. Disponível em: $<$ https://pubmed.ncbi.nlm.nih.gov/31609772/>.

16. Glasgow JM, Zhang Z, O’Donnell LD, et al. Hospital palliative care consult improves value-based purchasing outcomes in a propensity score-matched cohort. Palliat Med 2019 Apr;33(4):452-456. [acessado 2020 Jul 07]. Disponível em: $\langle\underline{\text { https://journals.sagepub.com/doi/10.1177/0269216318824270> }}$.

17. O'Mahony S, Johnson TJ, Amer S, et al. Integration of palliative care advanced practice nurses into intensive care unit teams. Am J Hosp Palliat Care 2017 May;34(4):330-334. [acessado 2020 Jul 07]. Disponível em:

<https://journals.sagepub.com/doi/abs/10.1177/1049909115627425>.

18. May P, Garrido MM, Cassel JB, et al. Palliative care teams' cost-saving effect is larger for cancer patients with higher numbers of comorbidities. Health Aff (Millwood) 2016 Jan;35(1):44-53. [acessado 2020 Jul 28]. Disponível em : <https://www.healthaffairs.org/doi/full/10.1377/hlthaff.2015.0752 >

19. May P, Garrido MM, Cassel JB, et al. Prospective cohort study of hospital palliative care teams for inpatients with advanced cancer: earlier consultation is 
associated with larger cost-saving effect. J Clin Oncol 2015;33(25): 2745-2752 [acessado 2020 Jul 30]. Disponível em: < https://ascopubs.org/doi/10.1200/JCO.2014.60.2334>.

20. Brinkman-Stoppelenburg A, Polinder S, Olij BF, van den Berg B, Gunnink N, Hendriks MP, et al. The association between palliative care team consultation and hospital costs for patients with advanced cancer: An observational study in 12 Dutch hospitals. Eur J Cancer Care (Engl) 2020 May;29(3):e13198. [acessado 2020 Jul 28]. Disponível em: <https://onlinelibrary.wiley.com/doi/epdf/10.1111/ecc.13198>. 